\title{
GROUPS WITH FINITELY MANY CONJUGACY CLASSES OF SUBGROUPS THAT ARE NOT NILPOTENT-BY-CHERNIKOV
}

\author{
HOWARD SMITH \\ Department of Mathematics, Bucknell University, Lewisburg, PA 17837, U.S.A.
}

(Received 19 July, 2005; accepted 4 March, 2006)

\begin{abstract}
We prove some theorems on locally graded groups and nilpotent-byChernikov groups.

2000 Mathematics Subject Classification. 20F18, 20F19, 20F50.

In paper [7] the authors established that if $G$ is a locally graded group in which every subgroup is nilpotent-by-Chernikov then either $G$ is nilpotent-by-Chernikov or $G$ is a perfect locally finite $p$-group for some prime $p$. In [1] it was pointed out that Theorem A of [7] contained an assertion about periodic groups that was not justified, but as the claim was only that the locally finite $p$-groups referred to above have all subgroups nilpotent, one sees that the following theorem is indeed a consequence of [7] and Theorem 1.3 of [1], an important result that deals with the case in which $G$ is a locally finite $p$-group. (It is clear from the Introduction in [1] that the author was quite aware of this consequence.)
\end{abstract}

THEOREM 1. Let $G$ be a locally graded group in which every subgroup is nilpotentby-Chernikov. Then $G$ is nilpotent-by-Chernikov.

We refer the reader to [1] for further references to articles along the lines of [7]. We remind the reader that a group $G$ is locally graded if every non-trivial finitely generated subgroup of $G$ has a non-trivial finite image; the above theorem presents a good indication as to why such a class of groups was introduced in the first place, as there exist infinite simple groups $G$ that have all proper non-trivial subgroups of prime order (see [8]). An apparently weaker hypothesis than a group's having all proper subgroups satisfying a certain property has been addressed in several articles, including [4], [11] and [12]. The hypothesis in question is that, for some property $\mathrm{P}$, the non-P subgroups of $G$ fall into finitely many conjugacy classes; in particular, Theorem 4.1 of [12] states that an infinite, locally graded group $G$ with just finitely many conjugacy classes of non-nilpotent subgroups is locally nilpotent and has only finitely many non-nilpotent subgroups. As was pointed out in [12], a non-nilpotent group with these properties is the direct product of a $p$-group $K$ and a finite $p^{\prime}$-group $F$, where $p$ is a prime, and $K$ (if nontrivial) has a "minimal non-nilpotent" subgroup $H$ of finite index. By Theorem 1.3 of [1] we have that $H$ is nilpotent-by-Chernikov, and so we may assert the following.

THEOREM 2. Let $G$ be a locally graded group and suppose that there are just finitely many conjugacy classes of non-nilpotent subgroups of $G$. Then $G$ has just finitely many non-nilpotent subgroups, and if $G$ is infinite and not nilpotent then $G$ is the direct product of a nilpotent-by-Chernikov p-group $K$ and a finite nilpotent $p^{\prime}$-subgroup $F$, for some prime $p$. In any case, $G$ is nilpotent-by-Chernikov. 
The main purpose of this note is to establish the following generalization of Theorem 1 . We shall see that most of the work in proving this has already been carried out.

THEOREM 3. Let $G$ be a locally graded group that is not nilpotent-by-Chernikov. Then $G$ has infinitely many non-(nilpotent-by-Chernikov) subgroups that are pairwise non-conjugate.

There is an interesting "bounded version" of Theorem 1, proved in [2]. This states that a locally graded group in which every proper subgroup is (nilpotent of class at most $n$ )-by-Chernikov ( $n$ a fixed positive integer) is (nilpotent of class at most $n$ )by-Chernikov. (Several results were established in [3] on groups in which all proper subgroups are (nilpotent (respectively, nilpotent of bounded class))-by-(finite rank), hypotheses that are in general weaker than those of being (nilpotent (or nil- $n$ ))-byChernikov. One consequence of Theorem 6 of that paper is that a locally finite $p$-group with all proper subgroups (nil-n)-by-Chernikov is soluble and nilpotent-by-Chernikov.) We remark that the case $n=1$ of the above theorem from [2] was successfully dealt with in [7], subsequent to the proof for $G$ periodic (and locally graded) in [9]. We shall denote by $N_{n} C$ the class of groups under discussion (and by $N C$ the class of nilpotent-by-Chernikov groups). Using this result from [2], our final theorem is a very easy consequence of Theorem 3 .

THEOREM 4. Let $n$ be a positive integer and let $G$ be a locally graded group that is not in the class $N_{n} C$. Then $G$ has infinitely many non- $\left(N_{n} C\right)$-subgroups that are pairwise non-conjugate.

The main requirement for our proof is the following lemma. Its proof is very similar to that of Lemma 4.5 of [12], and that proof in turn makes reference at one stage to the proof of Proposition 1 of [11]. Accordingly, we present a sketch proof only of our lemma, and invite the interested reader to supplement this sketch with the details from [11] and [12].

LEMMA. Let $G$ be a locally graded group with finitely many conjugacy classes of subgroups that are not nilpotent-by-Chernikov. Then $G$ is locally (soluble-by-finite).

Proof. Suppose the result false and let $S$ be a finitely generated subgroup of $G$ that is not soluble-by-finite. Denote by $R$ the finite residual of $S$. If $r$ is the maximal number of elements required to generate a finitely generated subgroup of $G$ that is not solubleby-finite (note that such an $r$ exists) then one shows that $S / R$ has rank at most $r$ and consequently is soluble-by-finite. The soluble radical $H / R$ of $S / R$ is not periodic, since it is infinite and finitely generated. Let $K$ denote the product of all normal subgroups of $S$ that are locally (nilpotent-by-finite) (or $L(N F)$, say); then $K$ is $L(N F)$, by the same argument as that used for establishing the Hirsch-Plotkin theorem (see, for example, $[10 ; 12.1 .2])$. Moreover, $K$ contains all of the subnormal $L(N F)$-subgroups of $S$. If $S / K$ is not soluble-by-finite and $H / M$ is an arbitrary soluble image of $H$ then $M$ is not $L(N F)$ and we may argue as in [12] to obtain a contradiction. So $S / K$ is solubleby-finite; let $J / K$ denote its soluble radical and let $U / K$ be the Fitting radical of $J / K$. If $u \in U \backslash K$ then $\langle u\rangle K$ is subnormal in $S$ and so is not $L(N F)$. The set of all such $\langle u\rangle K$ is a union of finitely many conjugacy classes and so $U / K$ is not periodic since it is infinite. We may therefore choose $x \in U$ that has infinite order modulo $K$. For each prime $p$ and positive integer $i$ the subgroup $X_{i}:=\left\langle x^{p^{i}}\right\rangle K$ is not $L(N F)$, and $K$ is the $L(N F)$-radical of $X_{i}$. Again we may follow the argument from [12] to obtain our final contradiction. 
Proof of Theorem 3. Again suppose that $G$ satisfies the hypotheses of the lemma. We shall show that $G$ is nilpotent-by-Chernikov, thereby establishing Theorem 3. If $G$ is $L(N F)$ (that is, locally (nilpotent-by-finite)) then $G$ satisfies the maximal condition locally and hence, by Lemma 3.2 of [4], satisfies the minimal condition for non$(N C)$-subgroups. Theorem 1 provides us with the result in this case. Assume for a contradiction that $G \notin L(N F)$, so that $G$ has a local system consisting of finitely generated non- $L(N F)$-subgroups. Every member of this local system is soluble-byfinite and hence, by the conjugacy class hypothesis, (soluble of derived length d)-by(order at most e) for some integers $d, e$ and, by Proposition 1.K.2 of [6], $G$ too has this structure, so $G$ is soluble-by-finite. Since the hypotheses on $G$ are inherited by subgroups of finite index, we may assume that $G$ is soluble.

Let $G$ be of minimal derived length subject to being non- $L(N F)$. If $G^{\prime}$ is not $L(N F)$ then $G / G^{\prime}$ has just finitely many conjugacy classes of subgroups and is therefore finite, so $G^{\prime}$ inherits our hypotheses and is $L(N F)$. By this contradiction $G^{\prime}$ is $L(N F)$ and so the $L(N F)$-radical $D$ of $G$ contains $G^{\prime}$. By the same argument as used for $G^{\prime}$ we see that every normal subgroup of $G$ that is not $L(N F)$ has finite index in $G$, and so $D$ is $L(N F)$ and $G / D$ is infinite. Furthermore, every subgroup that contains $D$ properly is not $L(N F)$ and therefore has finite index in $G$, so $G / D$ is infinite cyclic, say $G=D\langle g\rangle$. But, for each prime $p$, the subgroup $\left\langle x^{p}\right\rangle D$ is not $L(N F)$, and since there are infinitely many such subgroups and no two are conjugate in $G$ we have a contradiction that shows that $G \in L(N F)$ and hence completes the proof of Theorem 3.

Proof of Theorem 4. Suppose that $G$ satisfies the hypotheses but not the conclusion of Theorem 4. By Theorem 3, $G$ is nilpotent-by-Chernikov; in particular $G$ is $L(N F)$, and another application of Lemma 3.2 of [4], using the main result of [2] in place of Theorem 1 above, gives the desired contradiction.

\section{REFERENCES}

1. A. O. Asar, Locally nilpotent $p$-groups whose proper subgroups are hypercentral or nilpotent-by-Chernikov, J. London Math. Soc. 61 (2000), 412-422.

2. B. Bruno and F. Napolitani, A note on nilpotent-by-Cernikov groups, Glasgow Math. J. 46 (2004), 211-215.

3. M. R. Dixon, M. J. Evans and H. Smith, Groups with all proper subgroups nilpotentby-finite rank, Arch. Math. 75 (2000), 81-91.

4. S. Franciosi and F. de Giovanni, Groups satisfying the minimal, condition on nonsubnormal subgroups, in Infinite groups' 94 (de Gruyter, Berlin - New York, 1995), 63-72.

5. B. Hartley, Periodic locally soluble groups containing an element of prime order with Černikov centralizer, Quart. J. Math. Oxford Ser. (2) 33 (1982), 309-323. 1973).

6. O. H. Kegel and B. A. F. Wehrfritz, Locally finite groups (North-Holland, Amsterdam,

7. F. Napolitani and E. Pegoraro, On groups with nilpotent by Černikov proper subgroups, Arch Math. 69 (1997), 89-94.

8. A. Yu. Ol'shanskii, Geometry of defining relations in groups (Nauka, Moscow, 1989).

9. J. Otal and J. M. Peña, Groups in which every proper subgroup is Černikov-by-nilpotent or nilpotent-by-Černikov, Arch. Math. (Basel) 51 (1988), 193-197.

10. D. J. S. Robinson, A course in the theory of groups (Springer-Verlag, 1982).

11. H. Smith, Groups with finitely many conjugacy classes of subgroups of large derived length, Bollettino U.M.I. Ser. 7(A) 9 (1995), 167-175. 151.

12. H. Smith, Groups with few non-nilpotent subgroups, Glasgow Math. J. 39 (1997), 141- 